\title{
Depression, Ketamine Treatment, and the Theater of Consciousness
}

\author{
Michael A. Cerullo ${ }^{1, *}$, Andrew Stryker ${ }^{2}$ \\ ${ }^{1}$ Cincinnati Institute for Cognitive Science, USA \\ ${ }^{2}$ Greater Cincinnati Behavioral Health, USA
}

Copyright (C) 2016 by authors, all rights reserved. Authors agree that this article remains permanently open access under the terms of the Creative Commons Attribution License 4.0 International License

\begin{abstract}
In severe depression thoughts of suicide can develop over weeks or months. Traditional pharmacologic or psychotherapeutic treatments take several weeks before they begin to improve symptoms. This gradual improvement is consistent with our everyday experience of the self. However, a new treatment, ketamine, can alter depressive symptoms within hours or even minutes. Ketamine can stop suicidal thoughts in as little as 20 minutes and is being developed to treat suicidal patients in the emergency room. Rapid changes in a belief as complex as suicide raise new questions about the self and identity. More complicated models of the self are required to understand how rapid changes in complex beliefs are possible without invoking unrealistic specificity in the brain (i.e. grandmother cells for belief). If the brain has multiple competing selves within modules then ketamine could work by either turning a self module on or off. This is consistent with a modified version of the global workspace theory of consciousness where a self module provides the context for the spotlight of attention.
\end{abstract}

Keywords Consciousness, Ketamine, Depression, Global Workspace, Self

\section{Introduction}

Depression is the most common psychiatric illness and occurs in over $15 \%$ of the population. ${ }^{1}$ The etiology of depression is unknown and depression is defined by a group of symptoms and their time course. ${ }^{2}$ Common symptoms include sad mood, anhedonia, insomnia, fatigue, hopelessness, and cognitive problems. In severe depression thoughts of suicide can develop as symptoms worsen and are the most common reason for hospital admission. Suicidal thoughts typically develop after weeks or months of serious depression. Traditional pharmacologic or psychotherapeutic treatments take three or four weeks to begin to improve symptoms. ${ }^{3,4}$ This gradual improvement of mood and cognition is consistent with our everyday experience of the self. However, a new treatment, ketamine, a dissociative anesthetic, can alter depressive symptoms within hours or even minutes. ${ }^{5-10}$ Ketamine can stop suicidal thoughts in as little as 20 minutes and is being developed to treat suicidal patients in the emergency room and allow them to return home and avoid hospitalization. ${ }^{7}$ Rapid changes in a belief as complex as suicide raise new questions about the self and identity. Typically, changes in complicated beliefs are caused by alterations in memory over time (i.e. experience). While simple beliefs can of course change permanently in minutes or even seconds (e.g. remember when you first learned whales are mammals and not fish), complicated beliefs are closely tied to identity and are typically resistant to rapid change. For example, we do not expect deep-rooted religious or political beliefs to change within minutes. The entrenched negative beliefs in depression are even more resistant to change and there is no known process (e.g. giving someone a million dollars) that can alter depression sooner than the standard psychotherapeutic or psychopharmacological treatments. Suicidal thoughts are part of an overriding negative view of the world that includes persistent beliefs that life is not worth living. Thus the near instant changes in these beliefs produced by ketamine seem to bypass the normal process whereby complicated beliefs change gradually over time.

\section{Ketamine}

Ketamine was first developed in 1962 as a general anesthetic. ${ }^{11}$ It is also classified as a dissociative anesthetic and can produce a sense of detachment and often hallucinations at lower subanesthetic doses. The mechanism of action of ketamine is through the blockade of glutamine binding sites on NMDA receptors. Glutamine is the primary excitatory neurotransmitter in the brain and NMDA receptors are found throughout the brain and are responsible for synaptic plasticity and memory formation. ${ }^{12}$ The action of ketamine is not specific to any brain region but appears to 
globally effect excitatory neurons. ${ }^{12}$

Similar to the discovery of the original tricyclic class of antidepressants, the efficacy of ketamine for depression was discovered serendipitously. Doctors were using subanesthetic doses to treat chronic pain and noticed improvement in mood. ${ }^{13}$ Berman et al. was the first study to examine the use of ketamine specifically for the treatment of depression. $^{5}$ Unlike other antidepressants, ketamine improves symptoms, including suicidal thoughts, within hours or minutes. ${ }^{7,8}$ To date there have been numerous open label trials of ketamine and it appears to be a promising treatment but large scale placebo controlled clinical trials are needed to determine if it can be used successfully to treat depression in standard clinical practice. ${ }^{5-10,14}$ Depression often returns within a few days of the first ketamine treatment and the larger clinical trials will help determine how many consecutive doses are required to sustain remission.

\section{The Case of Mr. J}

The case of Mr. J will help illustrate some of the philosophical challenges of ketamine treatment for depression and suicidal thoughts. ${ }^{15} \mathrm{Mr}$. J was a high functioning professional with a history of recurrent depression. He had been suffering from an episode of depression for the last several months that was refractory to traditional antidepressants. He continued to be able to work but had started to develop serious suicidal thoughts and he began to worry that he might act on them. Mr. J had seen an advertisement for a ketamine research study for suicidal thoughts and presented to the emergency room where the study was being conducted to receive the treatment. He was hoping to receive the treatment and then return to work latter that day.

Mr. J's story is not unique and many patients with depression often feel they have two selves: their depressed self and their normal self striving to return. When he presented to the emergency room Mr. J clearly had one self that wanted to get rid of the bothersome depression and suicidal thoughts and get on with his day. Let's call this self
$\mathrm{S}_{\mathrm{L}}$ (the self that feels Life is worth living). Yet another self was deeply depressed and was convinced that suicide was best solutions to his situation. We can label this self $\mathrm{S}_{\mathrm{NL}}$ (the self that feels it is Not worth Living). When he presented to the emergency room $S_{L}$ was clearly worried that $S_{N L}$ was going to win out and thus end the life of both selves. Unfortunately this is not uncommon in depression and psychiatrists in the emergency room make the call to admit patients to the hospital when they believe $\mathrm{S}_{\mathrm{NL}}$ is in charge.

\section{Ketamine Treatment}

Traditional treatments of depression do not challenge the way we think about changes in beliefs, and attitudes. Figure 1 shows the gradual change from beliefs that life is not worth living $\left(\mathrm{B}_{\mathrm{NL}}\right)$ over weeks into beliefs that life is worth living $\left(\mathrm{B}_{\mathrm{L}}\right)$ after treatment for depression. Figure 1 also shows how these high levels beliefs can evolve into the intermediary beliefs $\mathrm{B}_{\mathrm{NL} a}, \mathrm{~B}_{\mathrm{NL} b}$, and so on. For example, $\mathrm{B}_{\mathrm{NL} a}$ could involve having several beliefs about why life is worth living in addition to having some stronger beliefs that life is not worth living. This process could continue until the final belief $B_{L}$ is reached. These gradual changes are consistent with cognitive behavioral therapy and the incremental changes this therapy encourages as well as the incremental changes seen with antidepressant medications. ${ }^{3,16-20}$ However, the model in Figure 1 is clearly an extreme simplification. Beliefs about whether life is worth living are not isolated beliefs but instead are the end result of a large underlying belief network. Thus Figure 2 is a more accurate model of belief change in the brain. As can be seen in Figure $2, \mathrm{~B}_{\mathrm{L}}$ and $\mathrm{B}_{\mathrm{NL}}$ are part of a larger network of interrelated beliefs. These belief networks can change with time with $\mathrm{B}_{\mathrm{L}}$ evolving into $\mathrm{B}_{\mathrm{NL} a}, \mathrm{~B}_{\mathrm{NL} b}$, etc. as in Figure 1. Again this is consistent with common notions of beliefs, attitudes, and identity. With medications, neurovegetative symptoms usually improve first followed by a gradual improvement in mood. ${ }^{3,17,18}$ As the person's mood improves they begin to question their negative thoughts and beliefs that life is not worth living. Treatment response to psychotherapy also follows a similar timeline. ${ }^{16,18-20}$

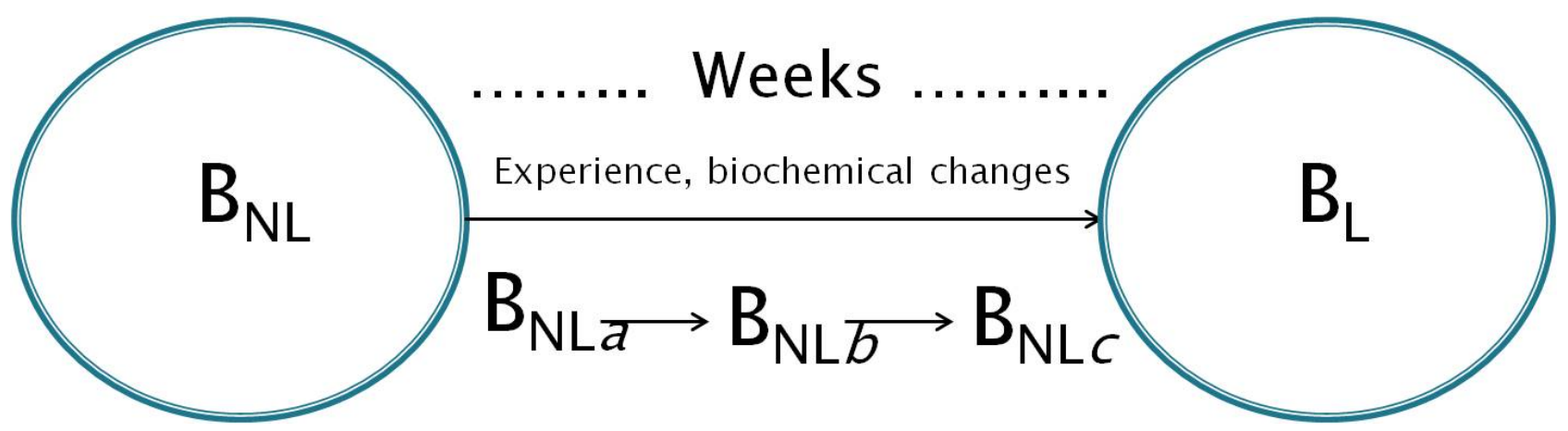

Figure 1. With traditional psychotherapeutic or psychopharmacological treatment of depression, beliefs that life is not worth living ( $\left.\mathrm{B}_{\mathrm{NL}}\right)$ change over several weeks into beliefs that life is worth living $\left(\mathrm{B}_{\mathrm{L}}\right)$. 


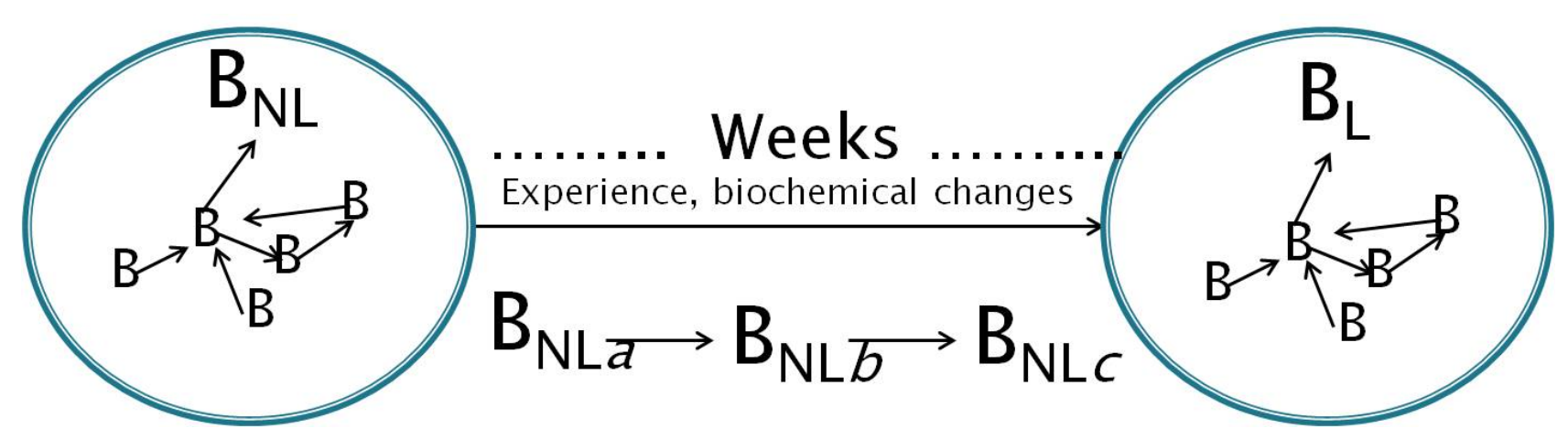

Figure 2. A more complicated model of belief change where high level beliefs such as $\mathrm{B}_{\mathrm{NL}}$ (belief that life is not worth living) and $\mathrm{B}_{\mathrm{L}}$ (beliefs that life is worth living) are part of a larger interconnected belief network. With traditional psychotherapeutic or psychopharmacological treatment of depression, beliefs that life is not worth living $\left(\mathrm{B}_{\mathrm{NL}}\right)$ change over several weeks into beliefs that life is worth living $\left(\mathrm{B}_{\mathrm{L}}\right)$ through gradual changes in the larger belief network.

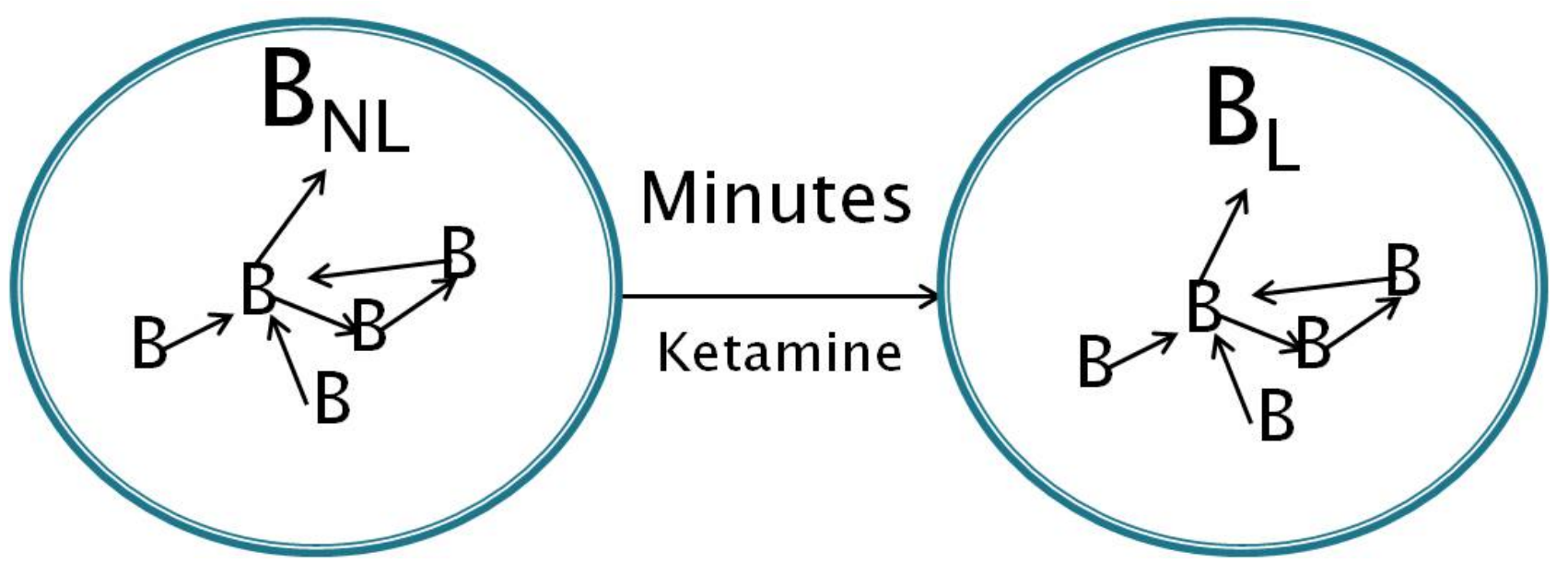

Figure 3. Ketamine causes rapid improvement (minutes) of depressive symptoms and suicidal beliefs and thus challenges traditional models of belief change.

Now we apply our treatment model to ketamine as shown in Figure 3. In this model $B_{N L}$ changes to $B_{L}$ within minutes. There is not time for high level beliefs to change as the end result of a gradual series of belief changes within the larger belief network. Instead, what seems to be happening is that high level beliefs like $\mathrm{B}_{\mathrm{NL}}$ are changed directly at the molecular level rather though intermediary beliefs. This would require the targeting of very specific cells by ketamine and would also imply the existence of grandmother cells for belief. The term grandmother cell was coined when a single cell in a group responding exclusively to pictures of participant's grandmother. ${ }^{21}$ Yet neuroscientists who study perception recognize that there is not any one cell responsible for such a high level perceptions. ${ }^{22}$ It seems even less likely that there would be a type of grandmother cell for $\mathrm{B}_{\mathrm{NL}}$. Thus the standard models of belief change do not work with Ketamine treatment.

\section{A New Model of the Self}

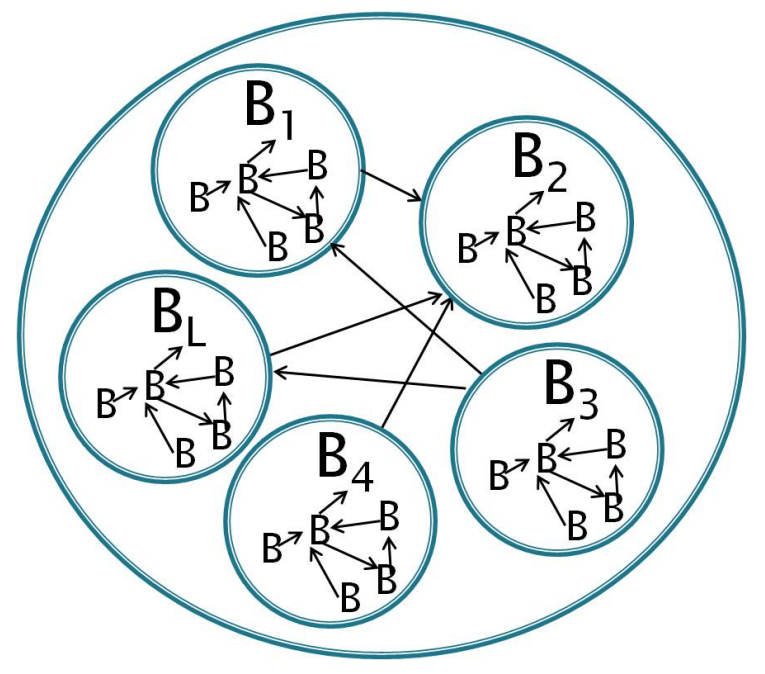

Figure 4. In order to understand Ketamine treatment a more complex model of the self is needed. In this model there are numerous self modules that cooperate and compete to produce the underlying attitudes and beliefs of the individual. 
The previous example illustrated the limitations of standard models of depression treatment when applied to ketamine. We therefore need to create a new model that allows rapid belief change in depression. Figure 4 illustrates a more complicated view of beliefs. As shown in Figure 4, the brain can be modeled by several independent modules that interact together to produce the attitudes and beliefs of the individual. One or more modules are responsible for high level extensional beliefs about whether life is (or isn't) worth living (we can call them the $\mathrm{B}_{\mathrm{NL}}$ and $\mathrm{B}_{\mathrm{L}}$ modules). This model is consistent with many modular theories of the brain. $^{23-25}$ However, other modular theories focus on individual modules for different perceptual or cognitive tasks which are often anatomically localized. The model we are proposing in Figure 4 is modular theory of the self. The area of influence of each self module need not be exclusive and there could be multiple modules that influence important existential or moral beliefs. The overall self may then emerge from a competition of influence among these modules and the influence of individual modules may wax and wane with time.

The existence of multiple self modules is consistent with Baars' global workspace model of consciousness. ${ }^{26,27}$ In the global workspace model, Baars uses the metaphor of a theater to understand consciousness. The brain can be thought of as the theater and attention is represented by a spotlight illuminating a specific part of the theater. When the attentional spotlight illuminates a particular part of the brain, that process reaches attention and enters consciousness. In Baars' model, brain regions don't wait passively for the spotlight but instead compete with each other and the region with the most influence draws the spotlight. Baars' model also includes what he calls unconscious contexts. These unconscious contexts are essentially models of the self with specific beliefs and values and could be identical to the different modules of the self we are proposing. In fact, Baars calls the underlying context the self workspace. ${ }^{26,27}$ In Baars' model, there was only one self but we can generalize his model to include multiple selves competing to be the underlying context for the global workspace. In this modified model there would be a second spotlight, a wide beam spotlight that provides a background illumination for the entire theater. The module of the self with the most influence then gets this illumination and provides the context for the narrow beam spotlight of attention (see Figure 5).

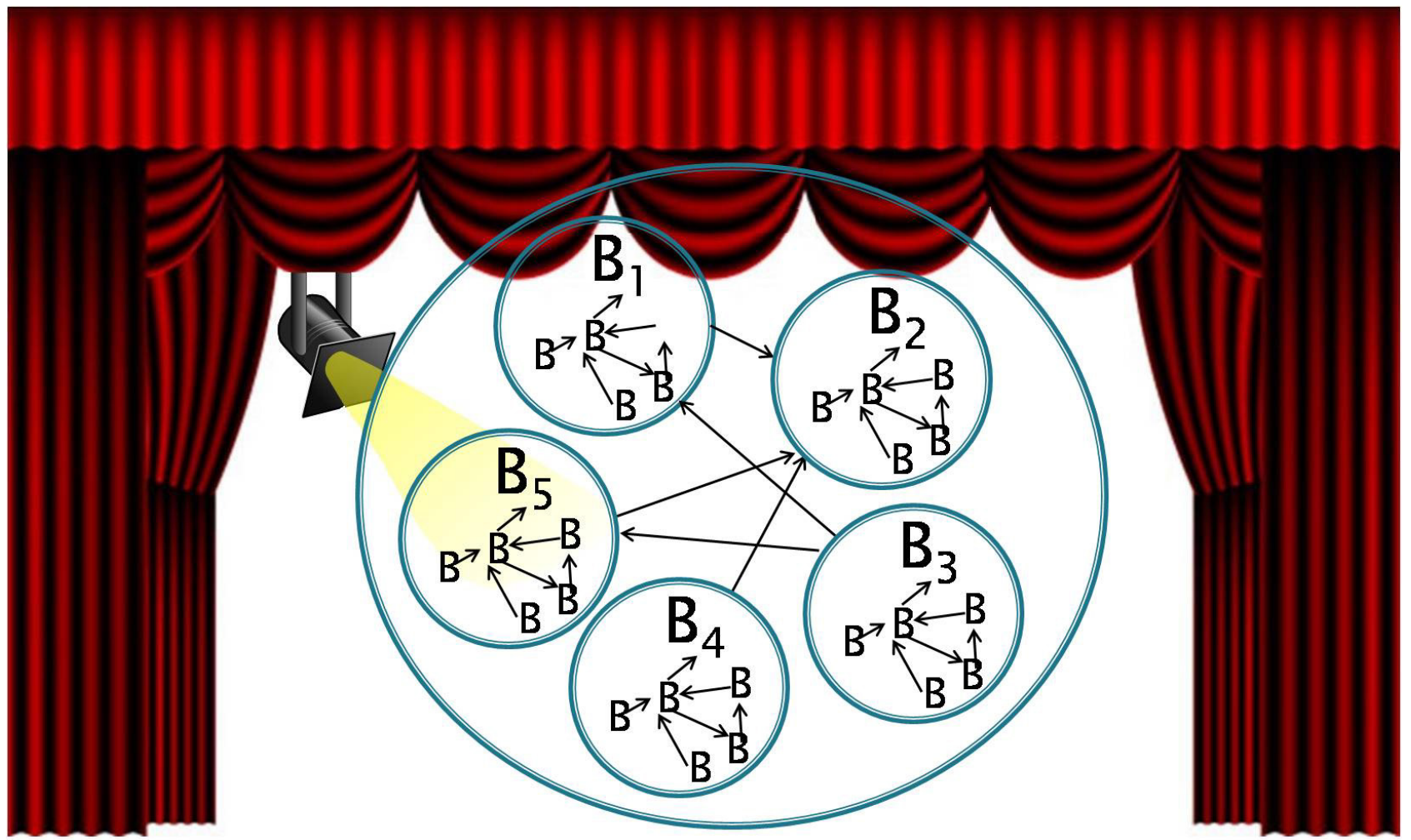

Figure 5. The modular theory of the self combined with Baars' global workspace model of consciousness. The different self modules $\left(\mathrm{B}_{1}\right.$ to $\left.\mathrm{B}_{5}\right)$ form the context for the theater of consciousness. The module of the self with the most influence (in this example $\mathrm{B}_{5}$ ) gets the focus of the wide beam spotlight and provides the context for the narrow beam spotlight of consciousness (the narrow beam spotlight is not shown in this diagram). 

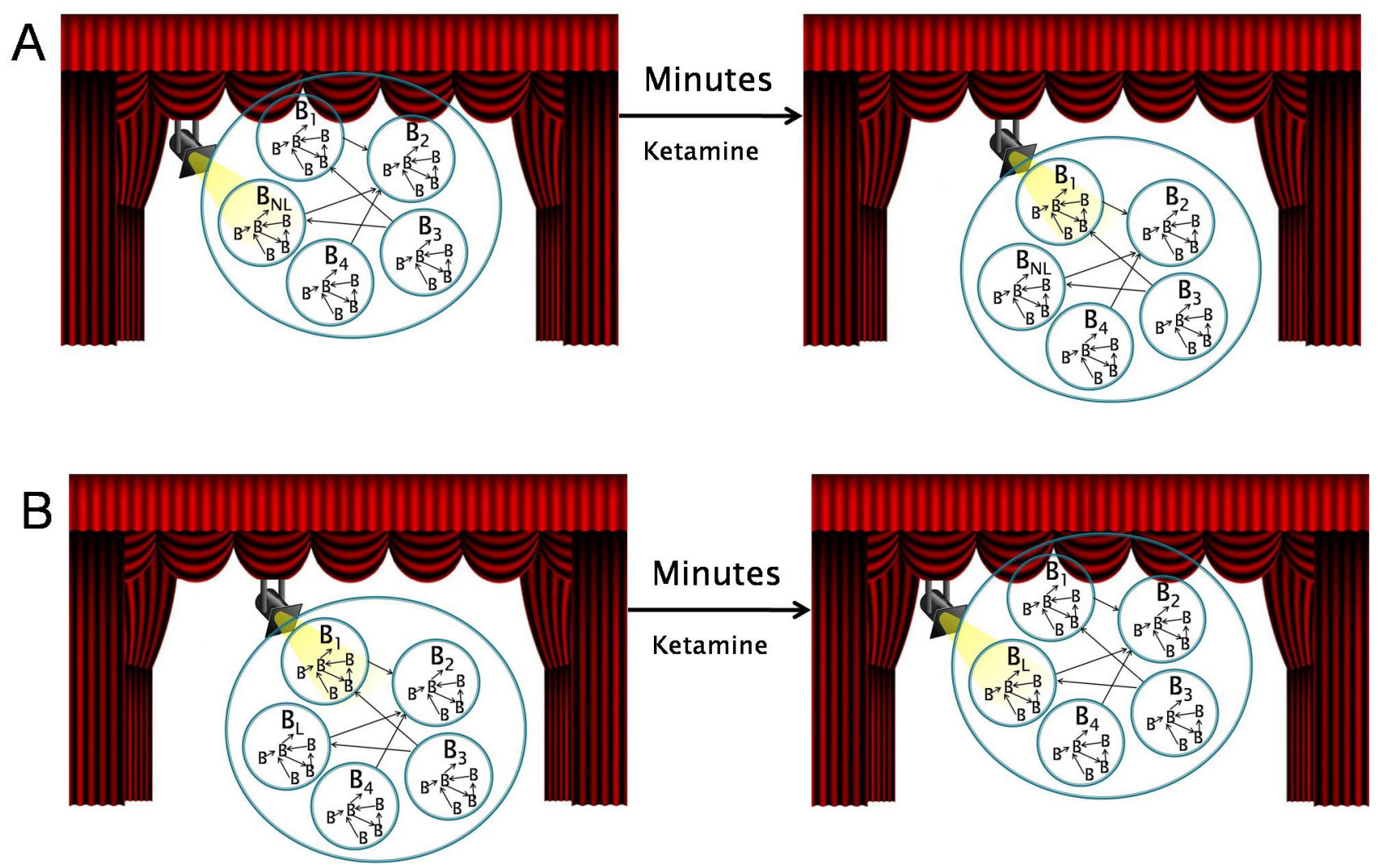

Figure 6. The complex modular model of the self along with global workspace can explain Ketamine treatment in two possible ways. Either ketamine is turning off the $\mathrm{B}_{\mathrm{NL}}$ module $(\mathrm{A})$ or turning on the $\mathrm{B}_{\mathrm{L}}$ module $(\mathrm{B})$.

\section{Ketamine and the Self}

We can now use a modular model of the self along with our modified global workspace model of consciousness to understand ketamine treatment. First, we can see that the new model is consistent with our prior treatment models of traditional antidepressants and psychotherapy. These long term therapies gradually change the modules responsible for the self and we do not require any new mechanisms to understand this process. In ketamine treatment, however, rapid changes in belief require a new mechanism. As discussed previously, ketamine does not target any specific region of the brain but could affect modules of the self that are distributed across the brain. Therefore ketamine could shift the broad beam spotlight of attention between different self modules. This broad beam spotlight could shift within minutes (the narrow beam attentional spotlight that can shift in a few hundred milliseconds) and thus explain the rapid changes in very complex beliefs. As can be seen in Figure 6, there are two possible mechanisms of action of ketamine in our new model. Ketamine could shift attention away from the $\mathrm{B}_{\mathrm{NL}}$ module and without the influence of this module other modules can again exert their influence that life is worth living and bring about a rapid change in suicidal thinking. The other potential mechanism of ketamine treatment would be that it focuses attention onto a specific $B_{L}$ module which then counters suicidal beliefs. Thus ketamine treatment could inactivate $\mathrm{B}_{\mathrm{NL}}$ or reactivate the $\mathrm{B}_{\mathrm{L}}$ module in minutes and thus treat depression rapidly.

Functional neuroimaging research is needed to better understand the neural mechanisms underlying rapid changes in complex mental states as well as the neural correlates of self modules. While our proposed model is currently speculative it could generate testable hypotheses in the future. For example, if neural correlates of various self modules are found in healthy participants, neuroimaging research could determine if these brain regions are altered with ketamine treatment. This research could ultimately be used to help develop better targeted treatments for depression in the future.

\section{Conclusions}

Large clinical trials currently underway will determine if ketamine can be used in large scale clinical practice. However, even our current limited experience with ketamine treatment shows that it is possible to rapidly change complex high level beliefs within minutes. Rapid changes in a belief as complex as suicidal ideation raise new questions about the self. More complicated models of the self are required to understand these changes without invoking unrealistic specificity of belief in the brain (i.e. grandmother cells for belief). If the brain has multiple competing modular selves then ketamine could work by either turning on or off a module via a mechanism similar to 
Baars' spotlight of attention, but on a much larger scale. Certain models of consciousness allow multiple simultaneous overlapping selves to be present in the normal brain. ${ }^{20,21}$ Ketamine treatment lends empirical support to such theories. In the near future rapid treatment of depression may be commonplace. More research is needed to understand the neural mechanisms underlying rapid changes in complex mental states and this knowledge may also require a rethinking of many aspects of the self.

\section{REFERENCES}

[1] Narrow W, Rae D, Robins L, Regier D. (2002). Revised Prevalence Estimates of Mental Disorders in the United States: Using a Clinical Significance Criterion to Reconcile 2 Surveys' Estimates. Achieves of General Psychiatry. 59, 115-123.

[2] American Psychiatric Association. (2013). Diagnostic and statistical manual of mental disorders (5th ed.). Arlington, VA: American Psychiatric Publishing.

[3] Quitkin F, Rabkin J, Ross D, Stewart J. (1984). Identification of true drug response to antidepressants. Use of pattern analysis. Arch Gen Psychiatry. 41, 782-6.

[4] Stahl, S. (2013). Stahl's Essential Psychopharmacology: Neuroscientific Basis and Practical Applications. New York, NY: Cambridge University Press.

[5] Berman R, Cappiello A, Anand A, et al. (2000). Antidepressant Effects of Ketamine in Depressed Patients. Biol Psychiatry. 47, 351-354.

[6] Zarate C Jr, Singh J, Carlson P, et al. (2006). A randomized trial of an N-methyl-D-aspartate antagonist in treatment-resistant major depression. Arch Gen Psychiatry. 63, 856-864.

[7] Larkin G, Beautrai A. (2011). A preliminary naturalistic study of low-dose ketamine for depression and suicide ideation in the emergency department. The International Journal of Neuropsychopharmacology. 14(8), 1127-1131.

[8] Covvey JR, Crawford AN, Lowe DK. (2012). Intravenous ketamine for treatment-resistant major depressive disorder. Ann Pharmacother. 46, 117-123.

[9] Murrough JW, Perez AM, Pillemer S, et al. (2013). Rapid and longer-term antidepressant effects of repeated ketamine infusions in treatment-resistant major depression. Biol Psychiatry. 74(4), 250-256.

[10] Kaplan, A. (2013). New Claims and Findings for Ketamine in Severe Depression. Psychiatric Times January 17. ePub. (http://www.psychiatrictimes.com/major-depressive-disorder /new-claims-and-findings-ketamine-severe-depression).
[11] Jansen, K.L. (2000). A Review of the Nonmedical Use of Ketamine: Use, Users and Consequences. Journal of Psychoactive Drugs. 32, 419-33.

[12] Kandel, E, Schwartz J, Jessell T. (2000). Principles of Neural Science. New York, NY: McGraw-Hill Medical; 4 edition.

[13] Correll G, Futter G (2006). Two case studies of patients with major depressive disorder given low-dose (subanesthetic) ketamine infusions. Pain Med. 7, 92-95.

[14] Diamond, P. et al. (2014). Ketamine infusions for treatment resistant depression: a series of 28 patients treated weekly or twice weekly in an ECT clinic. J Psychopharmacol. 28(6), 536-544.

[15] McCullumsmith, C. (2012). Ketamine Treatment. University of Cincinnati Department of Psychiatry Grand Rounds. Cincinnati, $\mathrm{OH}$.

[16] Beck, A. (1979). Cognitive Therapy and the Emotional Disorders. New York, NY: Penguin.

[17] Thibodeau M., et al. (2014). Latent Classes Of Nonresponders, Rapid Responders, And Gradual Responders In Depressed Outpatients Receiving Antidepressant Medication And Psychotherapy. Depression and Anxiety. 33(3), 213-220.

[18] Beck, J. (2011). Cognitive Behavior Therapy: Basics and Beyond. New York, NY: Guilford Press.

[19] Driessen E., Hollon S. (2010). Cognitive Behavioral Therapy for Mood Disorders: Efficacy, Moderators, and Mediators. Psychiatric Clinics of North America. 33(3), 537-55.

[20] Beck, J. S. (1995). Cognitive therapy: Basics and beyond. New York, NY: Guilford Press.

[21] Gross C. (2002). Genealogy of the "Grandmother Cell." Neuroscientist. 8(5), 512-518.

[22] Quiroga R, Kreiman G, Koch C, Fried I. (2008). Sparse but not 'Grandmother-cell' coding in the medial temporal lobe. Trends in Cognitive Sciences. 12(3), 87-91.

[23] Fodor, J. (1983). Modularity of Mind: An Essay on Faculty Psychology. Cambridge, MA: MIT Press.

[24] Baars, B. (1988). A cognitive theory of consciousness. New York, NY: Cambridge University Press.

[25] Sporns O. (2010). Networks of the Brain. Cambridge, MA: MIT Press.

[26] Baars, B. (1997). In the theater of consciousness: The workspace of the mind. New York, NY: Oxford University Press.

[27] Baars, B. (2005). Global workspace theory of consciousness: toward a cognitive neuroscience of human experience. Progress in Brain Research. 150, 45 -53. 